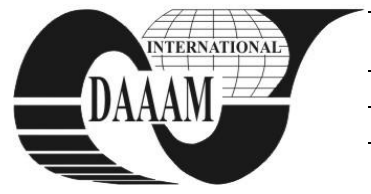

\title{
SENSOR FUSION FRAME WORK FOR MARBLE/STONE PROCESSING INDUSTRY USING LOW COST SENSORS
}

\author{
NAGLA, K[uldeep] S[ingh]; MOIN, U[ddin]; SINGH, D[ilbag] \& RATHI, N[ikhil]
}

\begin{abstract}
Multi sensor data fusion is highly demanding in fast growing stone production and process industries to cope with the large uncertainties in sensory information. Such uncertainties are very common due to information faliours, environment and sensor ambiguities. This paper presents sensor fusion framework by using low cost sensors to identify particular shape and size of marble slab sliding on the conveyor belt. The identification of particular shape of marble is required in applications such as stone grinding, cleaning, gluing and surface treatments etc. The information of heterogeneous sensors (IR and Sonar) is updated using Dempster Shafer theorem to reduce the uncertainties. The shape of the marble slab has been identified using qualitative comparision criterion.
\end{abstract}

Key words: robotics, sensor fusion, Dempster Shafer theory, IR and sonar sensors

\section{INTRODUCTION}

Object recognition is a significant requirement of high level autonomy in robotic applications. In several autonomous industrial applications researchers used various types of sensors and recognition techniques to estimate the shape of object around the manipulator (Selver, M.A., et al. 2009). Carved stones and marbles are used extensively for building construction and in particular for decoration purposed since ancient time. In the last three decades the market demands of carved and highly finished marble increases. Due to the technical advances in technology, especially in the fields of advanced digital image acquisition the production is increased but the manufacturing cost of vision sensors are still high (Sergio Saponara et.al, 2009) . This paper describes a combination of low cost sensors for stone factory automation. Sonar sensor is most widely used sensor in various robotics applications due to low cost, simple in operation and does not depend on brightness of the surface but it suffers from some serious problem as specular reflection, foreshortening, cross talking etc. IR sensor is another low cost sensor which is widly used for distance estimation in many robotics applications but it has its own limitations such as effect of high intensity ambient light on correct estimation of distance. In order to handle such problems sensor fusion is a suitable technique proposed to reduce the uncertainty in sensor measurement that is achieved by combining the sensory information from multiple sensors. In this particular application fusion is carried out by using Dempster Shafer theorem using DS occupancy grid. The experiment is conducted over dimensions of the $L$-shaped, $I$ shaped, $C$-shaped object (the $L, I$ and $C$ shapes have been considered for particular industrial application in which the large size objects i.e. marble slabs are sliding on the conveyor belt in front of the manipulator) for different applications.

\section{DESCRIPTION OF THE PROBLEM}

The aim of this work is to develop low cost an intelligent multisensor detection system with minimum ambiguity for the detection of objects passing on the conveyor belt in front of the manipulator. The following are the assumptions

\subsection{Assumptions}

- The target objects are translating on conveyor belt

- $n$-heterogeneous sensors are identifying the target geometry and each sensor observes the same area

- Each sensor model provides a two dimensional discrete grid-map and data is maintained relevant to current position of target in front of manipulator

- The manipulator performs application cleaning/grinding by predesigned manipulator according to selection of trajectory that depends upon the structural configuration of the target object.

- Three shapes of the object are considered (I-shape, Lshape and C-shape)

\subsection{System operation}

Let $\mathrm{n}$ numbers of sensors are fixed on the manipulator as shown in the figure 1 . The range estimation from different sensors are coneverted in to occupancy grid. A dempster shafer blief function has been used to evaluate the blief of every indivisual cell in the renge of the sensor. The DS grid based representation of environment has been selected due to advantage that it is not only represent occupied space but also represent the free space and don't know conditions of robot environment. The sensors ate fitted on the three DOF manipulator specially designed for cleaning applications. The current information gathered at time step $t$ of $i^{\text {th }}$ sensor denoted by $k_{i}^{t}$ where $i=1,2, \ldots, n$,. The Sonar and IR sensor occupancy grid is formulated by using Moravec-Elfes model of occupancy grid (Moravec, 1988). The occupancy grid is represented in the grid map configuration with $c_{i} \times d_{i}$ dimensions. At each step $t, i^{\text {th }}$ sensor represents the updated environment using Dempster shafer method in the form of local grid map. The fusion of information (from sonar and IR) is further performed and compared with reference model of known occupancy grid for particular shape. The shape of the sliding object is estimated by comparing the occupied and empty cells of 2D occupancy grid.

\section{DEMPSTER-SHAFER GRID MAP}

Dempster-Shafer theory represents evidence as a possibilistic belief function. DS theory represents the relevant characteristics of the world as a finite set of mutually exclusive propositions and assumptions called the frame of discernment (FOD). The possibilistic belief functions is also called Shafer belief functions, are combined used Dempster's rule of combination. The blief function is represents by occupancy, empty and don't know condition of indivisual cell. Thre results of DS theory are very similar to baysean theorem but it has an advantage to know don't know condition. Beore starting the updating of grid prior information 0.5 is considred in the case of the bays theorem but it is considred as 1 in case of DS theorem.

\section{MAPPING OF ENVIRONMENT USING SONAR SENSORS}

Grid mapping is commonly used to map the environment in mobile robots. We proposed this method to investigate the 
shape of the object moving on the conveyor belt for particular application. The grid mapping is first proposed by Elfies (Elfies, 1987) in wich the environment is convered in the tessellation of $n$ number cells. By using elfis model the occupancy and emptyness of every indivisual cell can be evaluated in the form of probability. Later the probabilistic model can be updated by several ways like using bays theorem, DS theorem and fuzzy inference etc. we use Dempster Shafer grid map that splits the grid in to three parts (using sonar) i.e. sonar occupied $\left(\right.$ sonar $_{\mathrm{o}}$ ), sonar empty $\left(\operatorname{sonar}_{\mathrm{e}}\right)$, and sonar disjunctive ( sonar $_{\mathrm{o}, \mathrm{e}}$ ) i.e., don't know, according to equations (1), (2) and (3). The similar model is used for IR sensor.

$m_{i, j}^{(o)}=m_{i, j}^{(o) e m} \oplus m_{i, j}^{(o) s}$
$=\frac{m_{i, j}^{(o) e m} m_{i, j}^{(o) s}+m_{i, j}^{(o) e m} m_{i, j}^{(o, e) s}+m_{i, j}^{(o, e) e m} m_{i, j}^{(o) s}}{1-m_{i, j}^{(e) e m} m_{i, j}^{(o) s}-m_{i, j}^{(o) e m} m_{i, j}^{(e) s}}$

$m_{i, j}^{(e)}=m_{i, j}^{(e) e m} \oplus m_{i, j}^{(e) s}$

(1)

$=\frac{m_{i, j}^{(e) e m} m_{i, j}^{(e) s}+m_{i, j}^{(e) e m} m_{i, j}^{(o, e) s}+m_{i, j}^{(o, e) e m} m_{i, j}^{(e) s}}{1-m_{i, j}^{(e) e m} m_{i, j}^{(o) s}-m_{i, j}^{(o) e m} m_{i, j}^{(e) s}}$

$$
m_{i, j}^{(o, e)}=m_{i, j}^{(o, e) e m} \oplus m_{i, j}^{(o, e) s}=\frac{m_{i, j}^{(o, e) e m} m_{i, j}^{(o, e) s}}{1-m_{i, j}^{(e) e m} m_{i, j}^{(o) s}-m_{i, j}^{(o) e m} m_{i, j}^{(e) s}}
$$

The terms $m_{i, j}^{(o) s}, m_{i, j}^{(e) s}$ and $m_{i, j}^{(o, e) s}$ are taken from sensor model as per the range detacted by sensor and $m_{i, j}^{(o) e m}, m_{i, j}^{(e) e m}$ and $m_{i, j}^{(o, e) e m}$ are taken from existing grid map (initial condition of every indivisual cell is considred as 1, i.e don't know condition)

\section{EXPERIMENTAL SETUP}

The apparatus shown in figure 1 consists of roller conveyer belt and 3-DOF manipulator. The objects (marble slabs) are sliding on the roller conveyer belt in front of the manipulator. The manipulator is fitted with two pairs of distance sensors. Sonar and IR sensors are placed over each other to estimate the distance between the sliding slab and the sensor position. Different shapes (I, L and C) are used to investigate the results of sensor fusion.

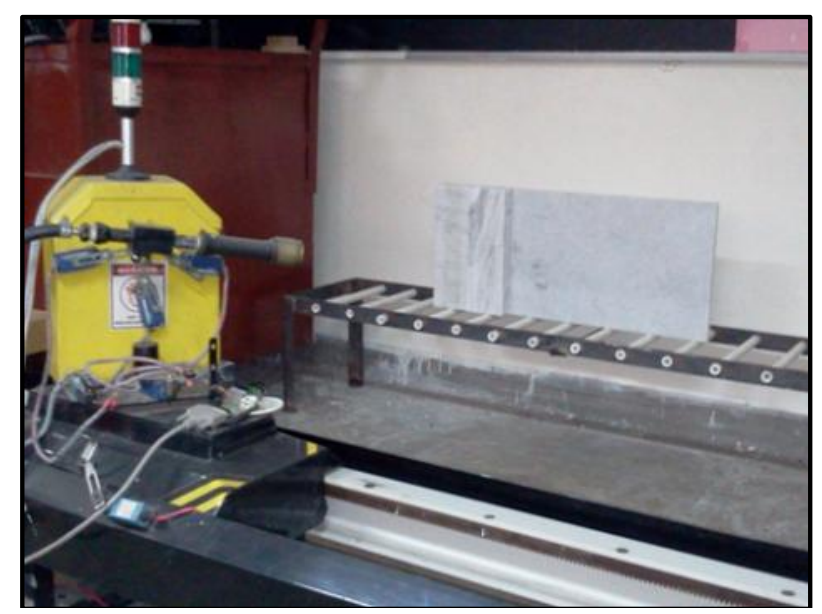

Fig. 1. Roller conveyor belt and 3-DOF manipulator fitted with various sensors

\section{RESULTS}

The experiment conducetd for $\mathrm{L}, \mathrm{I}$ and $\mathrm{C}$ shape marble slabs. The $2 \mathrm{D}$ and $3 \mathrm{D}$ occupancy grid is construced by updating DS model. A quantitative comparision criterion QCC is used to evaluate and compare the performance of mapping approach quantitatively.

$$
\frac{\mathrm{QCC}}{100}=\frac{\# \text { of correct occupied cell in the map being evaluated }}{\# \text { of all occupied cells in the map being evaluated }}
$$

Where a correct occupied cell is designated occupied in both reference map and map being evaluated.

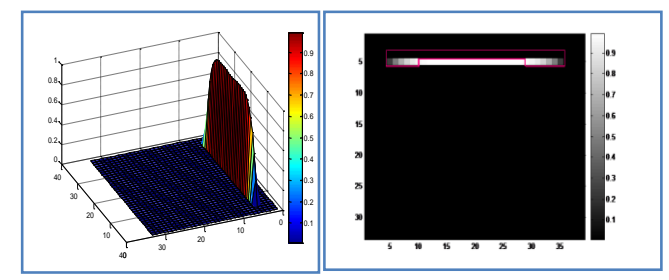

(a)

(b)

Fig. 2. (a) 3D representation of occupied region and (b) 2-D representation of Gray scale representation of fusion of two sonar sensor for $\mathrm{C}$-shaped object (White cells show maximum occupancy).

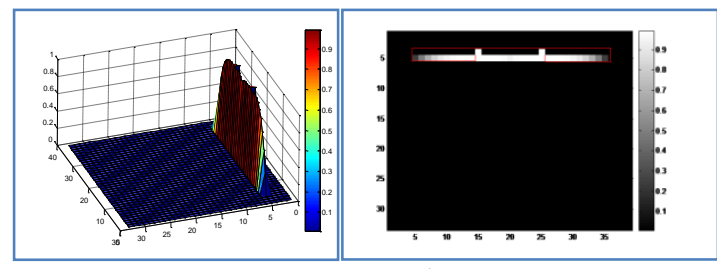

(a)

(b)

Fig. 3. (a) 3-D representation of fused occupancy grid (b) 2-D Gray scale representation of fusion of two sonar and two IR sensor for C-shaped object showing the removal of uncertainty generated by homogenious sensors sonars

\section{CONCLUSIONS}

The main contribution of this work is to detect object on conveyer belt and to identify the uncertainties generated by single sensor. The uncertainty generated by single sensor such as sonar or IR is reduced by fusing the sonar sensory information with IR sensory information using Dempster's rule of combination.

Figure 2 (a) and (b) shows the simulation results that the estimation of $\mathrm{C}$ type object by using two sonar sensors is inaccurate. However using the same sensors the estimation of I and $\mathrm{L}$ shape object was correct. The results in figure 3 (a) and (b) show that the fusion of sonar and IR has led to reduction of uncertainty in estimation of $\mathrm{C}$ type object. This technique provided useful information for detecting of the objects for optimal trajectory slection for said application. Future work may be extended by considering other sensor fusion techniques such as fuzzy logic algorithms and Neural Network for similar estimations.

\section{REFERNCES}

H. P. Moravec, "Sensor Fusion in Certainty Grid for Mobile Robots," AI magazine, vol. 9, 1988, pp. 61-74

Alberto Elfes, "Sonar-Based Real-World Mapping and Navigation", IEEE Journal of Robotics and Automation, Vol.3, No. 3, June 1987, pp 249-265

Sergio Saponara , Fabrizio Iacopetti, "Capacitive Sensors for process Control in Industrial Marble Machines" , IEEE International Workshop on Intelligent Data Acquisition and Advance Computing Systems, September 2009, pp 21-23

Selver, M.A.; Akay, O.; Ardali, E.; Yavuz, A.B.; Onal, O.; Ozden, G.; Cascaded and Hierarchical Neural Networks for Classifying Surface Images of Marble Slabs , Systems, Man, and Cybernetics IEEE Transactions Volume: 39, Issue: 4 : 2009 , pp 426 - 439 